\title{
ARTICLE \\ Cues play a critical role in estrous cycle-dependent enhancement of cocaine reinforcement
}

\author{
Amy R. Johnson ${ }^{1,2}$, Kimberly C. Thibeault ${ }^{2,3}$, Alberto J. Lopez ${ }^{1,2}$, Emily G. Peck ${ }^{4}$, L. Paul Sands ${ }^{4}$, Christina M. Sanders ${ }^{5}$, \\ Munir Gunes Kutlu' ${ }^{1}$ and Erin S. Calipari (iD ${ }^{1,2,3}$
}

While preclinical work has aimed to outline the neural mechanisms of drug addiction, it has overwhelmingly focused on male subjects. There has been a push in recent years to incorporate females into existing addiction models; however, males and females often have different behavioral strategies, making it important to not only include females, but to develop models that assess the factors that comprise female drug addiction. Traditional self-administration models often include light or tone cues that serve as discriminative stimuli and/or consequent stimuli, making it nearly impossible to disentangle the effects of cue learning, the cues themselves, and acute effects of psychostimulant drugs. To disentangle the interaction between drug-associated cues and the consummatory and appetitive responding driven by cocaine, we have developed a new behavioral procedure that combines Pavlovian-instrumental transfer with behavioral economic analysis. This task can be completed within a single session, allowing for studies looking at estrous cycle stage-dependent effects in intact cycling females, something that has been difficult in the past. In this study, we found no differences in self-administration across the estrous cycle in the absence of cues; however, when cues were introduced, the cues that acquired value during estrus-but not during diestrus or in males-increased motivation. Cues paired during estrus also increased c-fos expression to a greater extent in striatal regions, an effect that may underlie the observed increases in seeking induced by these cues, even weeks later. Together, these data suggest that fundamental differences in the motivational properties of psychostimulant drugs between males and females are complex and are driven primarily by the interaction between drug-associated stimuli and drug effects.

Neuropsychopharmacology (2019) 44:1189-1197; https://doi.org/10.1038/s41386-019-0320-0

\section{INTRODUCTION}

A major goal of preclinical addiction work is to understand the biological basis of drug addiction with the goal of improving treatment outcomes. Epidemiological evidence suggests that females represent a particularly vulnerable population [1]. Clinical studies have shown that women transition from first drug use to addiction faster, have more difficulty remaining abstinent, and have greater cue-induced cravings than men [2-5]. These factors have been recapitulated in rodent models [6-13] suggesting that the differences in humans are likely due to biological factors $[2,14-16]$. Going forward, it will be imperative to use preclinical models to define the factors that contribute to enhanced vulnerability to addiction in females.

Research in behavioral pharmacology has outlined the complex nature of reinforcement, highlighting the critical roles that schedules, context, and cues play in this process [17-21]. The interaction between cues and motivation is critical in the case of sex differences, as women exhibit increased cue responses, including craving [22-24]. Further, the subjective effects of cocaine and craving change over the menstrual cycle, highlighting a role for hormonal cycles in the process $[23,24]$. While in primates the menstrual cycle is approximately 28 days in length, in rodents a similar hormonal cycle-termed the estrus cycleoccurs on a shorter time span, approximately 4 days. Similar to primates, in rodents, cocaine effects are increased during estrus $[9,12,25]$ when circulating ovarian hormones are elevated $[2,6]$. In addition to altering the pharmacodynamic properties of cocaine, recent work [26] showed that the cycle stage when Pavlovian associations between cocaine and contextual cues were established played a critical role in the expression of conditioned behavior. This is important, especially in the context of motivated behaviors, as Pavlovian stimuli can enhance reinforced responding through a process called Pavlovian-instrumental transfer [27, 28]. Traditional self-administration procedures often include cues, making it nearly impossible to disentangle the effects of cue learning, the cues themselves, and acute effects of drugs. Understanding these interactions is particularly critical in forming effective interventions for addicted individuals.

Here we define how estrous cycle stage interacts with cues to influence motivation in intact, cycling females. We developed a new behavioral procedure that combines Pavlovian-instrumental transfer with behavioral economics to isolate the effects of cues on the consummatory and appetitive responses driven by cocaine. We find robust cycle-dependent changes in Pavlovian learning.

\footnotetext{
'Department of Pharmacology, Vanderbilt University School of Medicine, Nashville, TN 37232, USA; ${ }^{2}$ Vanderbilt Center for Addiction Research, Vanderbilt University School of

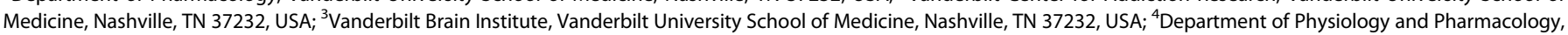
Wake Forest School of Medicine, Winston-Salem, NC 27101, USA and ${ }^{5}$ Neuroscience Department, Vanderbilt University School of Medicine, Nashville, TN 37232 , USA Correspondence: Erin S. Calipari (erin.calipari@vanderbilt.edu)

These authors contributed equally: Amy R. Johnson, Kimberly C. Thibeault.
}

Received: 22 August 2018 Revised: 5 January 2019 Accepted: 13 January 2019

Published online: 23 January 2019 
A
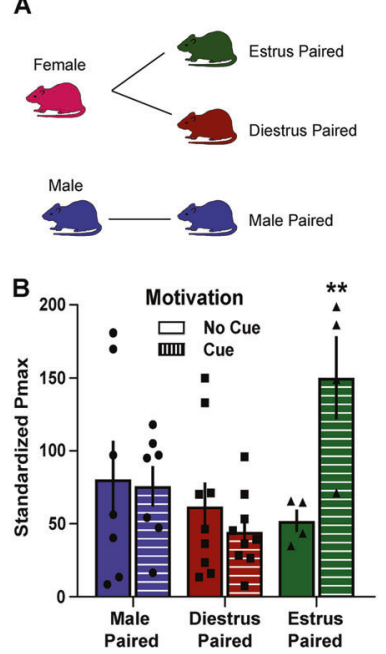
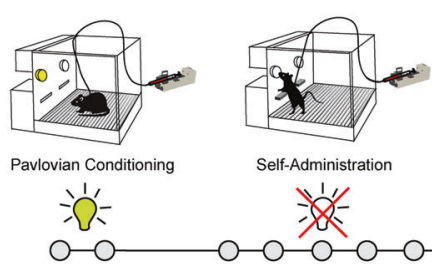

$\mathrm{O}-\mathrm{O}$

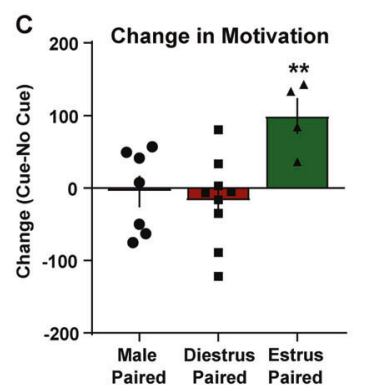

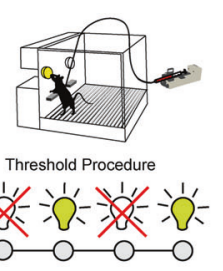

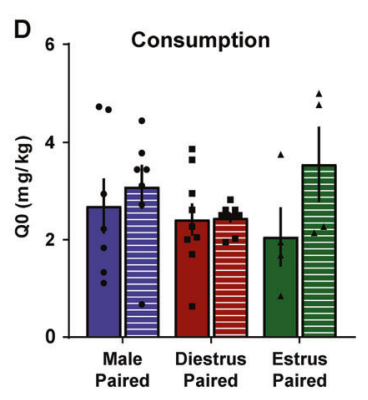

E
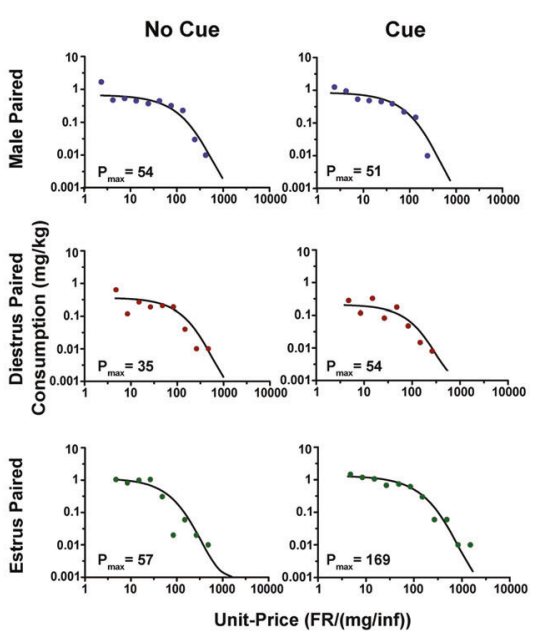

Fig. 1 Estrus females form associations between cocaine and predictive cues that enhance motivation later. a Timeline of experiments. Animals were first given non-contingent cocaine $(0.3 \mathrm{mg} / \mathrm{kg} / \mathrm{inj})$ on a VT300s schedule paired with a cue light ( $n=4-9$ per group). Next animals acquired self-administration in the absence of cues. Finally, the threshold procedure was used to assess consummatory and appetitive responding in the presence or absence of cues. b Estrus-paired cues increased motivation to obtain cocaine as compared to diestrus-paired or male rats. ${ }^{* *} p<0.01$ compared to no cue. c Animals were more motivated to self-administer cocaine in the presence of estrus-paired cues. There was no effect of cues in diestrus-paired or male animals, showing that the cue value, not just the presence of a cue light, is the critical determinant of changes in motivational drive. ${ }^{* *} p<0.01$ compared to male and diestrus females. $\mathbf{d}$ Cocaine intake did not differ between groups with cue or without. e Representative demand curves showing cocaine consumption as a function of price in males and females with or without cues-data is based off of the cycle state in which the cues were paired. All data are presented as mean \pm S.E.M

Pairing sessions with low-dose cocaine were capable of forming strong Pavlovian associations in estrus females and subsequently increasing motivation for cocaine independent of the cycle stage during self-administration. These cues activated ventral striatal regions, which correlated with drug-seeking weeks following the last self-administration exposure. These data show that cycle stage during self-administration is not the only factor determining enhanced cocaine motivation in females. Importantly, we show sex differences in vulnerability can be driven by interactions between cycle stage, cocaine effects, and cues.

\section{METHODS}

Animals

Adult male and female Sprague-Dawley rats purchased from Envigo (Indianapolis, IN) were maintained on a 12:12 h reverse light/dark cycle (0700 h lights off; $1900 \mathrm{~h}$ lights on). Animals were food restricted to $95 \%$ of free-feeding weight; males were between $325-450 \mathrm{~g}$ and females between $250-275 \mathrm{~g}$ at the beginning of studies. All animals were pair-housed for the duration of the experiments. All experiments were conducted in accordance with the guidelines of the Institutional Animal Care and Use Committee at Vanderbilt University School of Medicine, which approved and supervised all animal protocols.

\section{Surgical procedures}

Rats were anesthetized with ketamine $\left(100 \mathrm{mg} \mathrm{kg}^{-1}\right)$ and xylazine $\left(10 \mathrm{mg} \mathrm{kg}^{-1}\right)$ and implanted with chronic indwelling jugular catheters as described previously $[29,30]$. All sessions took place during the active/dark cycle (1200-1500 h). All animals were allowed at least two days of recovery from surgery prior to behavioral training.

\section{Apparatus}

Rats were trained and tested daily in individual Med Associates (St. Albans, Vermont) operant conditioning chambers fitted with a horizontal array of two retractable levers with cue lights above each lever. In each experiment, one of the levers (right or left) was the active lever and the other the inactive lever for the duration of the experiment and lever placement was counterbalanced throughout the experiment. Animals were connected through a tether to a single-speed syringe pump to allow for intravenous (IV) infusion of cocaine through the catheter either in response to an active lever press or non-contingently (see below for different conditions).

\section{Experimental timeline}

The experimental timeline for each experiment is outlined in each figure (Figs. 1a, 2a, 3c, 4b). Animals were first placed into a Pavlovian conditioning session where non-contingent cocaine injections occurred in conjunction with the presentation of a cue light or unpaired session where the cue light presentations and cocaine injections occurred in separate sessions (Phase 1). Subsequently, animals acquired self-administration in the absence of cues until they reached acquisition criteria (Phase 2). Once the rats acquired cocaine self-administration, threshold testing began (Phase 3). Each subject ran on the threshold procedure four times, twice without cues accompanying the cocaine injections and twice with the cue light accompanying cocaine injections. Female subjects ran one cue and one no-cue session once in estrus (proestrus/estrus) and once in diestrus (diestrus1/diestrus2) (Fig. 4a). The order of these sessions was counterbalanced between subjects. Finally, animals went through cue testing where they were allowed to lever press for the presentation of Pavlovian cues in isolation at 1 day withdrawal and again at 30 days (Phase 4).

Phase 1a: Pavlovian pairings

After surgery and recovery, rats were placed into an operant box with levers retracted and given 12 IV injections of cocaine $(0.3 \mathrm{mg} /$ $\mathrm{kg}$ ) through their jugular catheter and paired with cue light presentation (Fig. 1a right). Non-contingent cocaine injections occurred on a variable time 300 schedule to avoid an interval learning effect. The cue light came on at the start of the cocaine infusion and remained on for $20 \mathrm{~s}$. This pairing session was repeated the next day for 2 sessions of cue + cocaine pairings and 24 total cocaine injections. Cycle stages for female rats were determined daily by vaginal swabbing and cytology. Proestrus was 

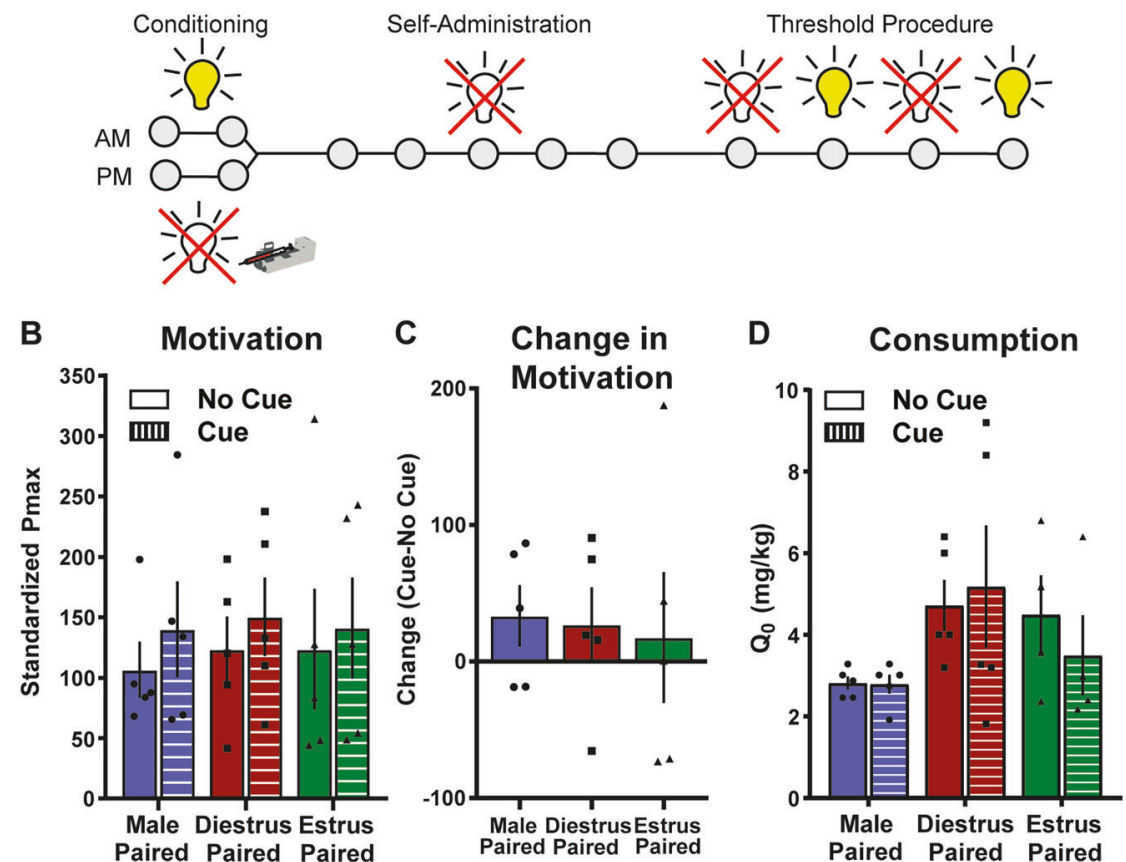

Fig. 2 Unpaired cues do not affect cocaine motivation. a Timeline of experiments. Animals were exposed to 12 presentations of the cue light on a VT300s schedule in the mornings and then in the afternoons given 12 cocaine injections on a VT300s schedule ( $n=5$ per group). Animals then acquired self-administration in the absence of cues. Finally, the threshold procedure was used to assess consummatory and appetitive responding in the presence or absence of cues. $\mathbf{b}$ Unpaired cues do not affect motivation to self-administer cocaine, showing that the pairing of the cue + the cocaine injection during estrus is the key determinant of the increased motivation to take cocaine. c There were no differences between groups in the change in $\mathrm{P}_{\max }$ caused by unpaired cues. $\mathbf{d}$ Cocaine intake did not differ between groups or between cue conditions. All data are presented as mean \pm S.E.M

defined as a predominance of nucleated epithelial cells, estrus was defined as a predominance of cornified epithelial cells while diestrus was defined as the predominance of leukocytes (see ref. [31] for detailed methods). The subjects were then divided into groups based on the stage they were in during the pairing sessions. One group had cues paired during proestrus and estrus (termed estrus-paired throughout the manuscript for simplicity) and the other in diestrus 1 and diestrus 2 (termed diestrus-paired) (Fig. 1a).

Phase 1b: unpaired condition

After surgery and recovery, separate groups of rats were placed into an operant box with levers retracted and given 12 cue light presentations (Fig. 2a). The rats were removed from the boxes and $2 \mathrm{~h}$ later, placed back into the boxes and given $12 \mathrm{IV}$ injections of cocaine $(0.3 \mathrm{mg} / \mathrm{kg})$ through their jugular catheter with no cues present. Both cue light presentations and cocaine injections occurred on a variable time $300 \mathrm{~s}$ schedule as in the Pavlovian pairing group. These sessions were repeated the next day for 2 sessions of cues and 2 session of cocaine injections for a total of 24 total cue presentations and cocaine injections.

Phase 2: self-administration training

Following the Pavlovian pairing sessions, rats were placed back into the operant boxes for self-administration training. Sessions were $2 \mathrm{~h}$ in length and presses on the active lever were reinforced by a $0.8 \mathrm{mg} / \mathrm{kg}$ infusion of cocaine followed by a $20 \mathrm{~s}$ timeout. In this phase cocaine injections were not paired with any exteroceptive cue such as a light or lever retraction. Animals were trained under these conditions until animals responded $>70 \%$ on the active lever and made $>20$ responses. Rats met the criteria in an average of $6.51( \pm 0.81)$ days of training with no significant differences between groups. Following acquisition animals moved onto the threshold procedure to assess estrous cycle-dependent changes in consummatory and appetitive responding in the presence or absence of the previously paired Pavlovian cues.

\section{Phase 3: threshold procedure}

The threshold procedure is a behavioral economics approach to assess drug taking/seeking and reinforcing efficacy [32, 33]. It consists of giving rats access to reinforcer while increasing the price, in effort, an animal must pay to obtain the reward. For the procedure with cocaine a descending series of 11 unit doses of cocaine $(421,237,133,75,41,24,13,7.5,4.1,2.4$, and $1.3 \mu \mathrm{g} /$ injection for males; the dose was cut in half for females) are available on an FR1 schedule of reinforcement with no timeout. Each dose is available for $10 \mathrm{~min}$, with each bin presented consecutively across the 110-min session. During this time, the lever is never retracted, and the only time out periods occur during the infusion of cocaine. By plotting the consumption of cocaine as a function of price (number of responses need to obtain $1 \mathrm{mg}$ of cocaine) demand curves are generated and behavioral economic principles can be applied to assess a variety of economic measures (described in detail below). During the initial bins of the procedure, the dose is high, thus minimal effort is needed to obtain a preferred level of drug, i.e., the cost of cocaine is low. With decreasing doses the cost progressively increases and animals are required to exert more effort to obtain their preferred level of cocaine. The point where the cocaine level is no longer maintained (i.e., the first derivative point slope of the function $=-1$ ) is termed the $P_{\max }$ or the maximal price paid. $P_{\max }$ is directly correlated with traditional measures of reinforcing efficacy like progressive ratio responding [30, 32]. Shifts in responding across the demand curve can be analyzed using behavioral economics principles, as described below. Responding during the first bin of the procedure is considered to reflect a loading phase and is not included in the analyses. 
A
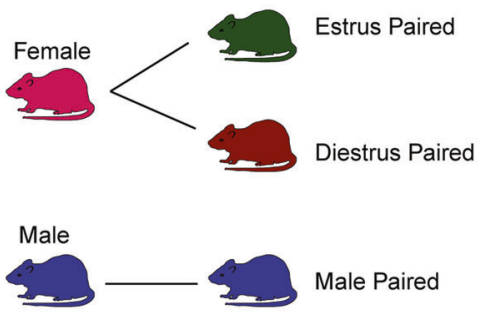

B

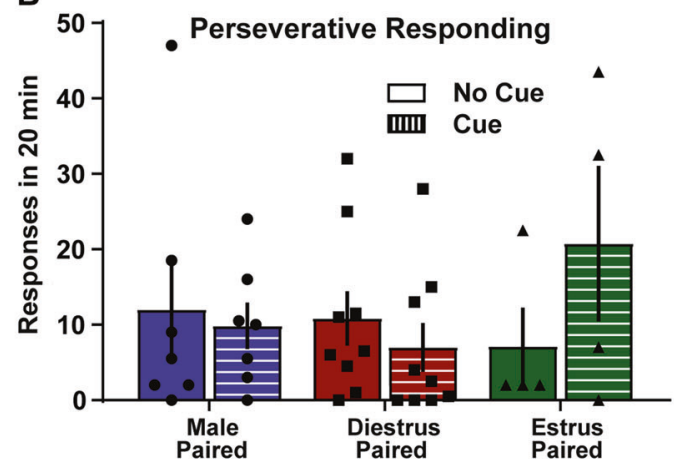

C

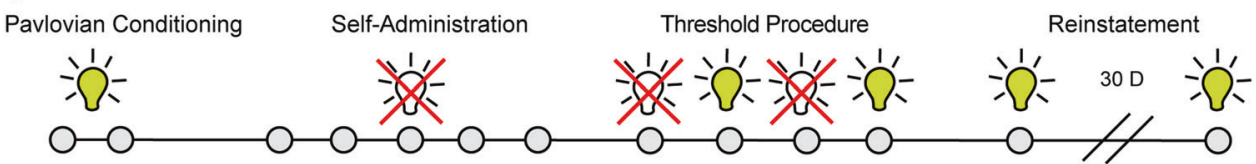

D

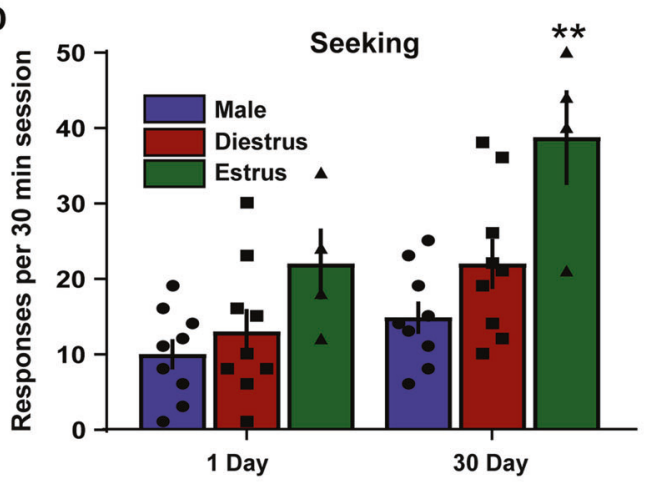

E

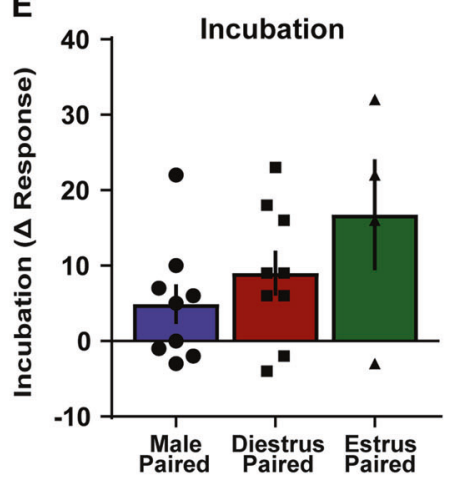

Fig. 3 Rats will lever press for cues paired with cocaine during estrus. a Experimental groups: cues paired during estrus, during diestrus, or in males ( $n=4-9$ per group). b There was no effect of cue pairing stage on perseverative responding. c Timeline of experiments for incubation testing. d Animals were placed into the operant chamber and allowed to lever press for the presentation of a cue light one day and 30 days following self-administration. Estrus-paired cues engendered higher rates of responding at day $30 .{ }^{* *} p<0.01$ compared to male and diestruspaired groups. e Incubation was not different between any of the groups. All data are presented as mean \pm S.E.M

Behavioral economic analysis was used to determine the parameters of maximal price paid $\left(\mathrm{P}_{\max }\right)$ and consumption at a minimally constraining price $\left(\mathrm{Q}_{0}\right)$, as described previously $[32,34$, 35]. Briefly, $P_{\max }$ and $Q_{0}$ values were derived mathematically using a demand curve. Demand curves were generated by curve-fitting individual animals' intake using an equation: $\log (Q)=\log \left(Q_{0}\right)+$ $k \times\left(e-a \times Q_{0} \times C-1\right)[36,37]$. In this equation, $P_{\max }$ was determined to be the unit price at which the first derivative point slope of the function $=-1$ [38]. The value $\mathrm{k}$ was set to two for all animals $[36,37]$.

$Q_{0}$. $\quad Q_{0}$ is a measure of the animals' preferred level of cocaine consumption. This can be measured when the dose is high and cocaine is available at low effort, or a minimally constraining price.

$P_{\text {max }}$. Price is expressed as the responses emitted to obtain $1 \mathrm{mg}$ of cocaine, thus as the dose is decreased in each consecutive bin of the threshold procedure, price increases. As the session progresses, animals must increase responding on the active lever in order to maintain stable intake. $P_{\max }$ is the price at which the animal no longer emits enough responses to maintain intake and consumptions decreases. Thus, animals with higher $P_{\max }$ will pay a higher price for cocaine. Previous work has demonstrated that $P_{\max }$ is highly correlated with break points on a progressive ratio schedule of reinforcement $[33,34]$.
Pavlovian-instrumental transfer sessions

Because the estrous cycle in a mouse occurs over a four-day period, traditional motivational tasks are not able to capture the full extent of motivation in this window as they require multi-day dose-response curves. Here we are able to capture the entire curve in a single day over each day of the cycle. This allowed for within subject assessment of consumption, motivation, and the effects of cues on these parameters over multiple estrous cycle stages. During testing, we were able to conduct four counterbalanced sessions: 2 where the cue was presented and 2 where no cue was presented. This allowed for the assessment of cues paired in estrus or diestrus with behavior done in estrus or diestrus. The design for each individual experiment is pictographically presented as a diagram at the top of each figure panel. Catheter patency was checked at the end of the study with Brevital $(5 \mathrm{mg} /$ $\mathrm{kg}$ ). Animals that did not have patent catheters were not included in the data analysis.

Phase 4: cue and incubation sessions

After the conclusion of the threshold sessions, rats were placed into the operant boxes and allowed to respond under a FR1 schedule of reinforcement for the presentation of the Pavlovian cue in the absence of cocaine. Lever pressing resulted in the presentation of the cue for $1 \mathrm{sec}$ and sessions were $30 \mathrm{~min}$ in length. Rats then underwent a 30-day withdrawal period during 
A
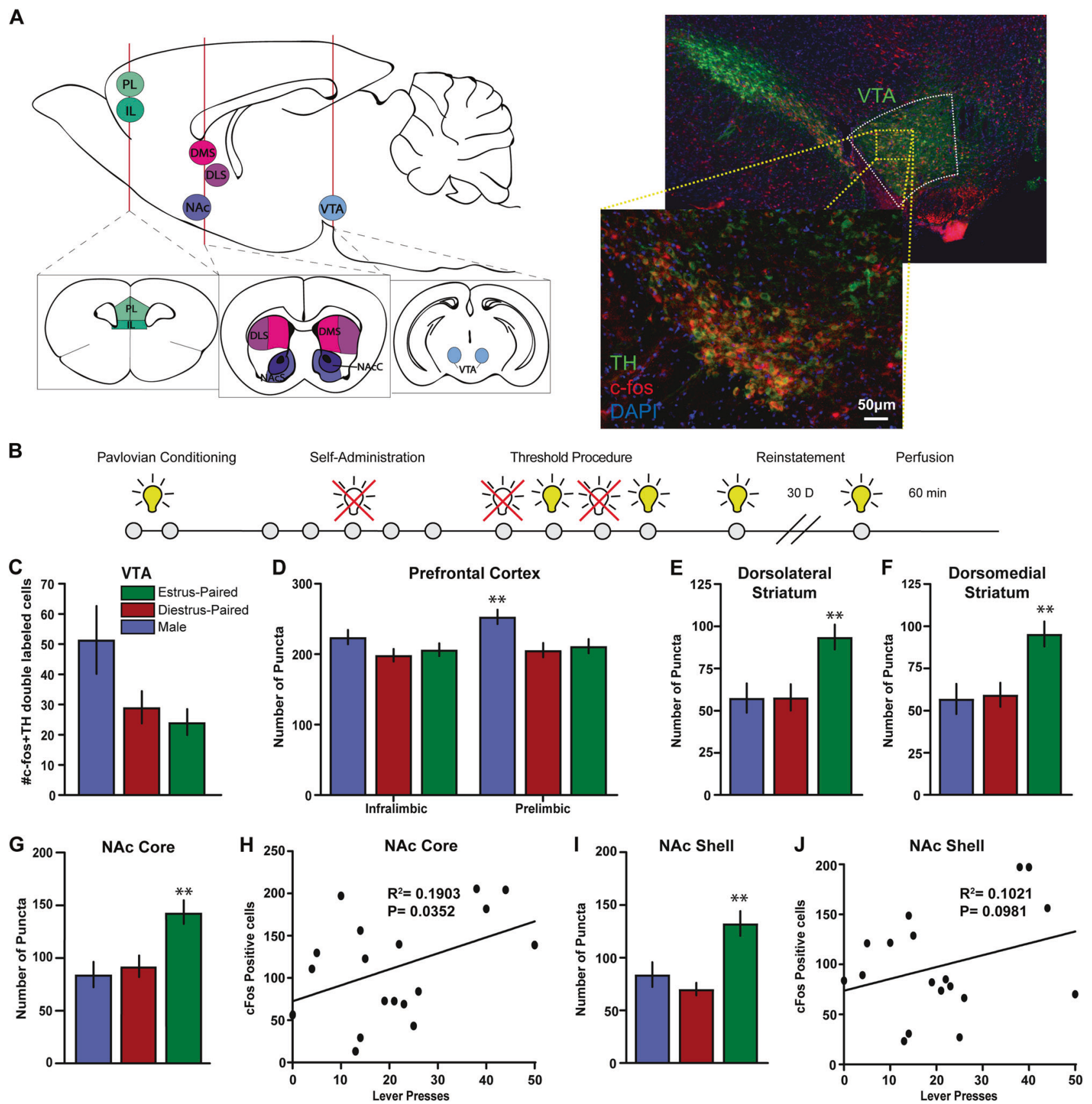

Fig. 4 Mesolimbic and mesocortical activation patterns that underlie enhanced cue effects on motivation. a Brain diagram depicting specific regions of interest. Assessment of activity, as measured by c-fos expression, was done in the prefrontal cortex (prelimbic and infralimbic regions), the dorsal striatum (dorsolateral and dorsomedial subregions), ventral striatum (nucleus accumbens core and shell) and the ventral tegmental area. (right) Representative image showing staining for $\mathrm{TH}+$ and c-fos + cells. b Timeline of experiments. c Number of c-fos + and $\mathrm{TH}+$ double-labeled cells in the VTA across groups. $\mathbf{d}$ c-fos + and NeuN+ double-labeled cells in the infralimbic and prelimbic regions of the prefrontal cortex, ${ }^{*} p<0.05$ compared to diestrus group (e) dorsolateral striatum, ${ }^{*} p<0.05$ compared to male group (f) dorsomedial striatum, (g) NAc core, ${ }^{*} p<0.05$ compared to male group and (i) NAc shell, ${ }^{*} p<0.05$ compared to diestrus-paired group. $\mathbf{h}$ Correlation between number of lever presses and number of double-labeled c-fos + and NeuN + cells in the NAc core or (j) NAc shell. All data are presented as mean \pm S.E.M

which they were in their home cage, received no cocaine, and did not run in any behavioral session. Previous studies have shown increases in responding for cocaine-associated cues after prolonged withdrawal, termed incubation [39] and this is seen as a measure of increased drug seeking due to incubation of craving. To see if the estrous cycle stage during cue and drug pairings had an effect on incubation, animals were placed back into the operant box on day 30 and performed another session where each lever press presented the Pavlovian cue to assess incubation of responding for the previously cocaine-paired cue. As animals in the unpaired condition never associated the cues with cocaine, these sessions were not performed in the unpaired groups.

c-fos staining as a measure of neuronal activation across the brain One hour following the last cue session, rats were transcardially perfused first with ice-cold PBS and then with a fixative solution containing 4\% PFA paraformaldehyde (PFA). Brains were postfixed for $24 \mathrm{~h}$ in $4 \%$ PFA at $4{ }^{\circ} \mathrm{C}$. Coronal sections of $30 \mu \mathrm{m}$ thickness were cut with a vibratome (Leica, Nussloch, Germany). Sections were permeabilized with $0.2 \%$ Triton X-100, followed by 
1194

blocking with bovine serum albumin. Primary antibodies against tyrosine hydroxylase (TH, Aves Labs, \#TYH, AB_10013440, 1:2000 dilution), c-fos (Millipore Sigma, \#ABE457, 1:1000 dilution) and NeuN (Millipore, \#MAB377, AB_2298772, 1:500) were diluted in blocking solution and sections incubated overnight at $4{ }^{\circ} \mathrm{C}$ with gentle shaking. Sections were then incubated with secondary antibodies [c-fos: donkey anti-rabbit AlexaFluor 594 AB_2340621 (\#711-585-152) and TH: donkey anti-chicken AlexaFluor 488 AB_2340375 (\#703-545-155); Jackson ImmunoResearch, 1:500 dilution] for $2 \mathrm{~h}$ at room temperature. After washing, sections were incubated for $5 \mathrm{~min}$ with DAPI (NucBlue, Invitrogen) to achieve counterstaining of nuclei before mounting in Prolong Gold (Invitrogen). Protein expression was assessed across the brain using a Keyence Digital Microscope (Keyence Corporation) imaged using $a \times 20$ or $\times 40$ objective with a 1.0 digital zoom. c-fos positive puncta were counted manually within each region.

The number of puncta per region was counted in: prefrontal cortex: infralimbic and prelimbic; nucleus accumbens (NAc): core and shell; dorsal striatum: dorsomedial and dorsolateral; and ventral tegmental area (VTA). In the VTA, only cells that were positive for both $\mathrm{TH}$ and $\mathrm{c}$-fos were counted. In all other regions, only cells that we co-labeled with NeuN and c-fos were counted. NeuN protein is localized in neuronal nuclei and this method ensured that the positive puncta being counted were neurons and not astrocytes or glial cells. In VTA, one slice per animal was counted, while in the other regions 3-5 slices per animal were counted. The number of cells for each region is plotted on each graph. For correlations, the number of positive neurons within each animal was correlated with the number of lever presses during the reinstatement session to determine the relationship.

\section{Statistics}

For the initial behavioral experiments, the number of lever presses were analyzed using a 2-way mixed model ANOVA (Cost $x$ Cue pairing stage) both with the cues and without the cues. The other behavioral parameters, standardized $P_{\max }$ and $Q_{0}$, were analyzed using 2-way mixed model ANOVA (Cue $x$ Cue pairing stage). Perseverative responding, reinstatement responding, and c-fos expression were all analyzed by one-way between subjects ANOVA. $P_{\max }$ values were further tested within estrus-paired, diestrus-paired and male groups between the cue and no cue sessions. We had an a priori hypothesis based on preexisting literature that the cues would increase $P_{\max }$ values, therefore we ran a one-tailed $t$-test. Type I error rate (alpha) was set to 0.05 for all statistical tests. Data is represented as the mean \pm S.E.M in figures.

\section{RESULTS}

Cues paired during estrus increase motivation for cocaine

We first aimed to determine how cues that acquired value during different stages of the estrous cycle could influence the motivational properties of cocaine. Pavlovian cues that acquired value during estrus increased motivation to self-administer cocaine as indicated by an increase in $\mathrm{P}_{\max }$ (Fig. 1b; 2-way mixed model ANOVA cue pairing stage $x$ cues interaction, $F_{(2,17)}=6.15$, $p=0.0098$ ). A similar pattern emerges with a difference score, calculated as the $P_{\max }$ during cue sessions minus the $P_{\max }$ during no cue sessions, with only the estrus group showing an increase in $P_{\text {max }}$ (Fig. 1c; 1-way ANOVA $F_{(2,17)}=6.11, p=0.01$ ). Previous work has shown that males can form associations that can potentiate self-administration [40], suggesting that these differences are not due to an inability of males and diestrus females to form associations.

Interestingly, the effects on motivation were dissociable from consumption. $Q_{0}$ was unchanged (Fig. 1d; 2-way ANOVA interaction, $\left.F_{(2,17)}=1.59, p=0.23\right)$, indicating that the cues influence motivation independent of other factors. Representative demand curves are shown in Fig. 1e.
Unpaired cues do not affect appetitive or consummatory responding for cocaine

Similarly to previous studies, in the unpaired groups, there were no differences in motivation (Fig. 2b; 2-way ANOVA, not significant) or consumption (Fig. 2d; 2-way ANOVA, not significant) between groups in the absence of cues. Further, unpaired cues did not have any effect on measures of motivation $\left(P_{\max }\right.$, Fig. $2 \mathrm{c}$; 2-way ANOVA, not significant) or consumption (Fig. 2d; 2-way ANOVA, not significant). These findings suggest that discrete paired cues are critical in the enhancement of cocaine motivation in this procedure.

\section{Cues do not affect perseverative responding}

Perseverative responding is defined by continued responding despite the absence of the reinforcing stimulus. The last two doses in the threshold task are non-reinforcing as animals will not selfadminister them in isolation. Previous studies have assessed perseverative responding during these epochs [33]. Perseverative responding did not differ between groups (Fig. 3b; 2-way ANOVA interaction, $\left.\mathrm{F}_{(2,17)}=1.99, p=0.17\right)$.

\section{Estrus-paired cues elicit more responding}

We next aimed to determine how Pavlovian cues influenced responding in the absence of drug. These sessions occurred one day after termination of cocaine self-administration and again 30 days later. There was no difference in responding for the cue on day one (Fig. 3d). All animals increased responding during the day 30 test session (Fig. 3d; 2-way ANOVA main effect test day $\left.F_{(1,19)}=21.25 p=0.0002\right)$ and the estrus-paired group responded more than either the male or diestrus paired group on day 30 (2-way ANOVA main effect pairing stage $\left.\mathrm{F}_{(2,19)}=8.89 p=0.0019\right)$. Incubation did not differ between groups when compared as a difference score (Fig. 3e; 1-way ANOVA $\left.\mathrm{F}_{(2,20)}=2.08 p=0.15\right)$.

Neural activation in the NAc core predicts responding for cues Next, we defined which brain areas are activated by the Pavlovian cues and whether this activation predicted behavior. One hour following the final cue session, animals were sacrificed and brains collected. Expression of c-fos, an immediate early gene, is used as a marker of activation. As the encoding of Pavlovian cues has been linked to mesolimbic and mesocortical dopamine systems, we focused on the ventral tegmental area (VTA), nucleus accumbens (NAc) core and shell, the dorsomedial and dorsolateral striatum. We also looked at c-fos expression in prefrontal cortical regions (infralimbic and prelimbic) that are involved in relapse and cue responding [41-43].

We found that c-fos expression $1 \mathrm{~h}$ after cue presentation in dopamine neurons in the VTA was affected by stage during cue pairing (Fig. 4c; 1-way ANOVA, $\left.\mathrm{F}_{(2,21)}=3.615 p=0.0447\right)$; however post-hoc tests did not reveal differences between groups. It is possible that greater dopamine release and activity resulted in feedback on the VTA cell bodies, causing extra variability within groups. This feedback has been seen previously in estrus females when dopamine agonists are administered [26]. Additionally, there was increased c-fos expression in areas downstream of the VTA, including the NAc core (Fig. 4g; 1-way ANOVA, $F_{(2,89)}=4.365$ $p=0.0155$ ), the NAc shell (Fig. 4i; 1-way ANOVA, $\mathrm{F}_{(2,85)}=3.589$ $p=0.0319$ ), and the dorsolateral striatum (Fig. 4e; 1-way ANOVA, $\left.\mathrm{F}_{(2,86)}=3.164 p=0.0472\right)$; however, $\mathrm{c}$-fos expression in the dorsomedial striatum was not significant although there was a trend towards significance (Fig. 4f; 1-way ANOVA, $F_{(2,86)}=3.067$ $p=0.0517)$. There was no effect of cycle stage at pairing on c-fos expression in the infralimbic cortex (Fig. 4d; 1-way ANOVA, $\mathrm{F}_{(2,85)}$ $=0.7516 p=0.4747)$. However, there was a difference in $c$-fos expression in the prelimbic cortex (Fig. 4d; 1-way ANOVA, $\mathrm{F}_{(2,85)}=$ $4.184 p=0.0185)$; although males had higher c-fos expression than diestrus females. It is important to note this finding as 
A

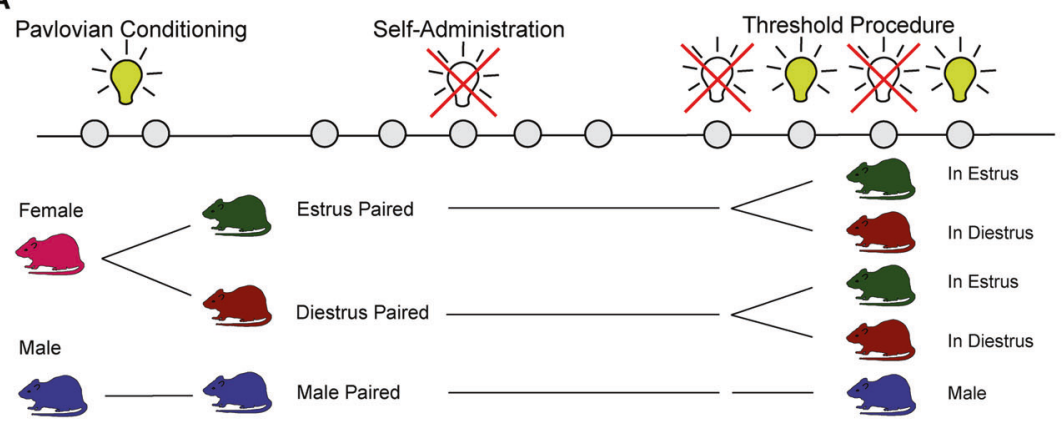

C

Estrous Cycle Stage on Test Day
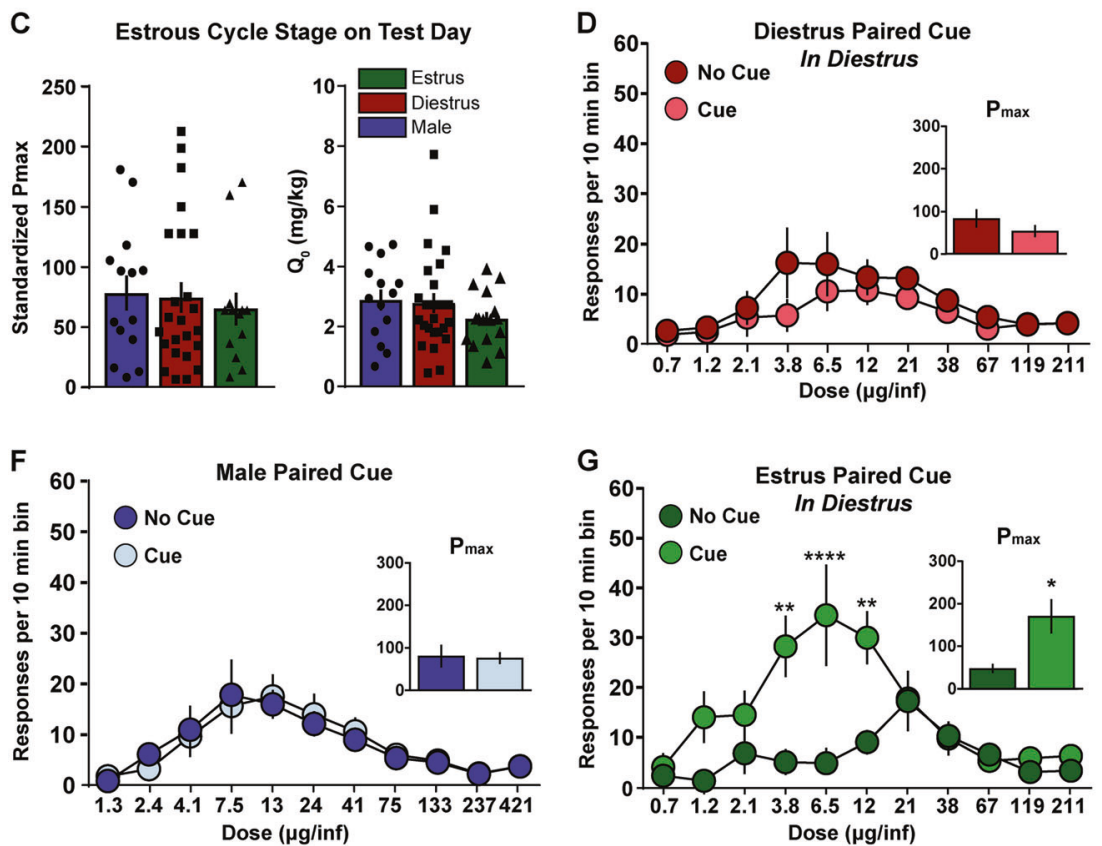

B

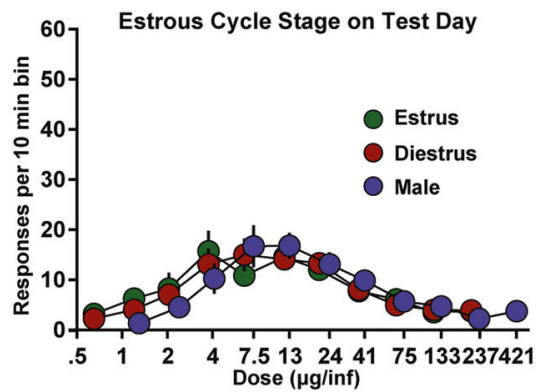

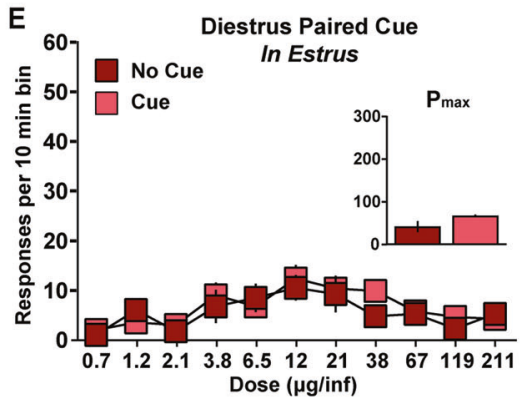

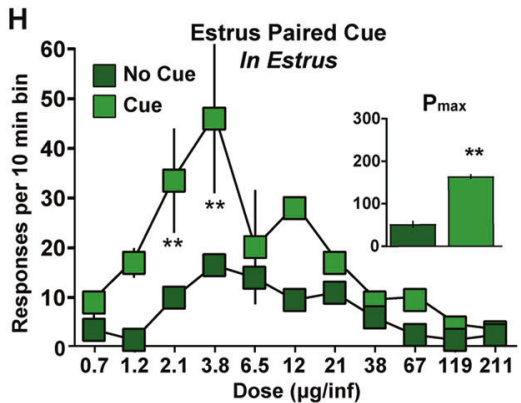

Fig. 5 Cues are critical in the expression of sex differences in motivation. a Timeline of experiments. Cues were paired during different cycle stages (estrus, diestrus, and in males) and then self-administration was conducted at different cycle stages (estrus, diestrus, males) to define the interaction between cue pairing stage and the cycle stage during self-administration $(n=4-9$ per group). b Self-administration responses based on cycle stage on test day. There were no differences in responses across the dose-response curve. c No effect of cycle stage on the day of testing for motivation as measured by $\mathrm{P}_{\max }$ (left) or $\mathrm{Q}_{0}$ (right). $\mathbf{d}$ There was no change in responding over the dose-response curve or in $P_{\max }$ (inset) in the presence of the diestrus paired cue when animals were in diestrus. e Similarly, there was no change in responding or in $P_{\max }$ (inset) when cue was paired during diestrus and animal was tested in estrus. $\mathbf{f}$ No change in responding or in $P_{\max }$ (inset) in the presence or absence of the cue in males. Cues that were paired in estrus increase motivation regardless of the cycle stage that the animal is in during testing. g Animals increased responding and $P_{\max }$ (inset) in the presence of the estrus-paired cue during diestrus. $\mathbf{h}$ Similarly, estrus-paired cues increased responding and $P_{\max }$ (inset) when animals were tested in estrus. For all panels ${ }^{* *} p<0.01,{ }^{* * * *} p<0.0001$ compared to no cue condition. All data are presented as mean \pm S.E.M

it suggests that not only are there estrous-cycle effects on cue-induced neuronal activation, but that there are also sexspecific differences that may not be controlled by ovarian hormones.

Together, these data suggest that in females, the estrous-cycle effects of cues driving motivated responding were predominantly localized to striatal regions. In order to determine if specific regional areas were more predictive of behavioral responses, we correlated the number of c-fos positive cells in each animal with their behavioral responding. We found a correlation between the number of c-fos positive puncta in the NAc core and lever pressing (Fig. $4 \mathrm{~h} ; \mathrm{R}^{2}=0.19 p=0.04$ ), suggesting that this area may play a role in cue effects on behavior. The correlation for the NAc shell showed a trend, but failed to reach significance (Fig. $4 \mathrm{j} ; \mathrm{R}^{2}=0.10$; $p=0.09$ ). Correlations in all other regions were not significant (data not shown).
Cues are critical for the expression of sex-differences in motivation Previous work has shown that hormonal factors can influence cocaine self-administration on a variety of behavioral tasks. However, many of these tasks contain cues that are paired with the delivery of the drug, thus, there are additional factors that could be contributing to these sex differences. Therefore, we aimed to determine whether cycle stage on test day or the cycle stage during the pairing was the critical factor contributing to motivational differences across the sexes. First, we wanted to determine if cycle stage - independent of cue presentation influenced self-administration (Fig. 5a). We found no effect of current cycle stage on the dose-effect curve (Fig. 5b; 2-way ANOVA interaction, $\mathrm{F}_{(20,590)}=0.81, p=0.71$; cycle stage main effect $\left.F_{(2,59)}=0.01, p=0.99\right)$. We also analyzed the data using economic principles and found no effect of current cycle stage on motivation (Fig. 5 c left; $P_{\text {max }}$ ) or consumption (Fig. $5 c$ right; $Q_{0}$ ). 
Next, we conducted experiments to determine whether cue pairing stage interacted with current cycle stage (Fig. 5a). There were 5 groups: male, diestrus paired cue currently in diestrus, diestrus paired cue currently in estrus, estrus paired cue currently in diestrus, and estrus paired cue currently in estrus. Each group was tested either in the presence or absence of the Pavlovian cues. When the cue was paired during diestrus and the animal was tested during diestrus there was not an effect on performance between the cue and no-cue conditions (Fig. 5d; 2-way ANOVA interaction, $\mathrm{F}_{(10,190)}=0.66, p=0.76$; cue main effect $\left.\mathrm{F}_{(1,19)}=1.02, p=0.32\right)$ or $P_{\max }$ (one-tailed $t$-test, $\mathrm{t}_{(13)}=1.11, p=0.14$ ). Similarly, when the cue was paired during diestrus, but the testing was done in estrus there was no effect of the cue on responding (Fig. 5e; 2-way ANOVA interaction, $\mathrm{F}_{(10,100)}=0.84, p=0.59$; cue main effect $\mathrm{F}_{(1,10)}=0.31$, $p=0.59$ ) or $\mathrm{P}_{\max }$ (one-tailed $t$-test, $\mathrm{t}_{(6)}=1.88, p=0.054$ ). Males did not show any differences between the cue and no-cue paring (Fig. 5f; 2-way ANOVA interaction, $\mathrm{F}_{(10,150)}=0.20, p=0.99$; cue main effect $\mathrm{F}_{(1,15)}=0.0006, p=0.98$ ) or $\mathrm{P}_{\max }$ (one-tailed $t$-test, $\mathrm{t}_{(12)}=0.16$, $p=0.44$ ). However, we found that the estrus-paired cues were capable of increasing motivation to self-administer cocaine in diestrus (one-tailed $t$-test $P_{\max }, t_{(6)}=2.92, p=0.013$ ) and in estrus (one-tailed $t$-test $\left.\mathrm{P}_{\max }, \mathrm{t}_{(2)}=11.4, p=0.004\right)$. Estrus-paired cues increased responding when the animal ran the threshold task in diestrus (Fig. $5 \mathrm{~g} ; 2$-way ANOVA cues $x$ cocaine dose interaction, $\mathrm{F}_{(10,60)}=5.44, p<0.001$ ), and in estrus (Fig. 5h; 2-way ANOVA interaction, $F_{(10,20)}=1.81, p=0.12$; cue main effect $F_{(1,4)}=2.48$, $p=0.0022)$, showing that predictive cues play a critical role in the sex differences in cocaine self-administration.

\section{DISCUSSION}

In this study, we define the powerful role that conditioned cues play in sex-differences in stimulant self-administration. Estrus females exhibit enhanced vulnerability to form associations between cocaine and Pavlovian cues that later influence volitional drug intake. During estrus, the associations formed between drugs and associated stimuli act to potentiate motivation, even months following initial pairing. Further, these cues alone can increase the activity of striatal regions, an effect that likely contributes to their enhanced motivational value. Together, these factors may combine to produce a unique state for females in which drugs and conditioned cues interact, thus enhancing addiction vulnerability and relapse. Understanding these interactions is critical, as it suggests that special attention needs to be given to effects of cues in female drug addicts.

The role that cues play in the expression of motivated behaviors between males and females is a critical gap in the current knowledge, as most addiction procedures are not designed to dissociate appetitive, motivational, and exteroceptive factors. Traditional self-administration models make it difficult to disentangle the effects of cue learning, cue effects, and drug effects; thus, we have developed a new procedure to specifically dissociate cue effects on discrete aspects of motivated behavior. Both cue pairing and motivational testing occur over 1-2 days, making this ideal for testing over the estrous cycle. In the absence of cues, estrous cycle stage had no effect on lever pressing, drug consumption, or motivation. However, when cues were present, the estrous cycle stage at which these cues acquired value was central to their ability to enhance motivation. Cues paired during estrus enhanced dopaminergic circuit activity, increased $P_{\max }$, and increased responding for these cues in the absence of drug. Together, these data suggest that fundamental differences in the motivational properties of drugs of abuse between males and females are complex and include interactions between drugassociated stimuli and drug effects.

While the lack of influence of cycle stage on motivation is inconsistent with the increases in progressive ratio responding that have been seen by previous groups $[12,25,44]$, these data suggest that cues may play a critical role in this process. However, it is also possible that differences in hormonal or estrous cycle effects on these self-administration tasks may represent a fundamental difference in what the task is measuring. Even though both progressive ratio and behavioral economic tasks are thought to measure motivation $[45,46]$, there are differences between these procedures that warrant discussion. In the case of progressive ratio, a defined dose is administered and animals must press a lever with increasing ratio requirements to continue self-administering. As the task progresses, fewer and fewer of the lever presses are reinforced, making it more difficult to maintain cocaine blood levels. Conversely, in threshold tasks, doses are decreased in successive bins; however, every lever press is reinforced, allowing for constant cocaine consumption, and maintenance of the animal's preferred blood level of drug. Thus, it is possible that estrous cycle effects on neural circuitry may act to maintain high levels of unreinforced responding, while not having any effect on the motivation to maintain cocaine levels/ consumption once consumption has begun. While there is some evidence that hormones influence progressive ratio responding, our findings align with other work using a behavioral economics procedure for cocaine self-administration in rats that found no effect of estrous cycle [47]. It is, however, important to note that in most cases $P_{\max }$ and progressive ratio break-points are correlated [32], suggesting other factors, such as cues, may be involved in the expression of these motivational differences.

We also aimed to pinpoint the neural circuits that underlie these effects. The ability to form associations between cues and rewards is dependent on activity of VTA dopamine cells as well activation in other reward-related brain regions such as the NAc $[27,48]$. This system is also critically involved in cocaine reinforcement [49-52]. Here we find that the activation of the nucleus accumbens and dorsolateral striatum, but not VTA or cortical areas, by Pavlovian cues was enhanced when those cues were paired during estrus and predicted cue-induced seeking. Together, these data show that cues paired during estrus result in greater activation of striatal brain regions and provides a mechanism by which cues could motivate self-administration behavior.

Overall, these studies show that sex differences in cocaine reinforcement are complex. Both clinical and preclinical literature have highlighted female subjects as a particularly vulnerable population, necessitating a complete understanding of the factors that contribute to this vulnerability. Here we outline an important role of cues in the enhancement of self-administration in females, demonstrating fundamental differences in the sex-specific regulation of reinforcement. Males and females often have different behavioral strategies, making it important not only to include females in current drug addiction models, but also to develop models that assess the factors that comprise female addiction. To this end, we have developed a new behavioral procedure that combines Pavlovian-instrumental transfer with behavioral economic analysis to disentangle the interaction between drugassociated cues and cocaine self-administration. The strength of this task is its short timeline, allowing for studies looking at estrous cycle stage-dependent effects in intact cycling females, something that has been difficult in the past. Together, this work provides a precise behavioral mechanism for the enhancement of motivation for cocaine in females and highlights the need for more complete behavioral studies dissociating discrete aspects of motivated behaviors between the sexes.

\section{FUNDING AND DISCLOSURE}

This work was supported by $\mathrm{NIH}$ grants DA042111 to ESC, MH065215-15 (ARJ) and MH064913-15 (KCJ) as well as by funds from the Brain and Behavior Research Foundation to ESC, the Whitehall Foundation to ESC, and the Edward Mallinckrodt Jr. Foundation to ESC. 


\section{ACKNOWLEDGEMENTS}

We would like to thank the NIDA drug supply program for providing the drugs used within this study. ARJ, KCT conceptualized studies, collected data, analyzed data made figures, wrote manuscript. EGP, LPS, analyzed data and edited the manuscript. AJL, CMS and MGK collected data, analyzed data, and edited the manuscript. ESC conceptualized studies, made figures, and wrote the manuscript.

\section{ADDITIONAL INFORMATION}

Publisher's note: Springer Nature remains neutral with regard to jurisdictional claims in published maps and institutional affiliations.

\section{REFERENCES}

1. Tuchman E. Women and addiction: The importance of gender issues in substance abuse research. J Addict Dis. 2010;29:127-38.

2. Becker JB, Hu M. Sex differences in drug abuse. Front Neuroendocrinol. 2008;29:36-47.

3. Chen K, Kandel D. Relationship between extent of cocaine use and dependence among adolescents and adults in the United States. Drug Alcohol Depend. 2002;68:65-85.

4. Randall CL, Roberts JS, Del Boca FK, Carroll KM, Connors GJ, Mattson ME. Telescoping of landmark events associated with drinking: a gender comparison. J Stud Alcohol. 1999;60:252-60.

5. Robbins SJ, Ehrman RN, Childress AR, O'Brien CP. Comparing levels of cocaine cue reactivity in male and female outpatients. Drug Alcohol Depend. 1999;53:223-30.

6. Jackson LR, Robinson TE, Becker JB. Sex differences and hormonal influences on acquisition of cocaine self-administration in rats. Neuropsychopharmacology. 2006;31:129-38.

7. Kerstetter KA, Aguilar VR, Parrish AB, Kippin TE. Protracted time-dependent increases in cocaine-seeking behavior during cocaine withdrawal in female relative to male rats. Psychopharmacol (Berl). 2008;198:63-75.

8. Lynch WJ, Roth ME, Carroll ME. Biological basis of sex differences in drug abuse: Preclinical and clinical studies. Psychopharmacol (Berl). 2002;164:121-37.

9. Lynch WJ, Arizzi MN, Carroll ME. Effects of sex and the estrous cycle on regulation of intravenously self-administered cocaine in rats. Psychopharmacol (Berl). 2000;152:132-9.

10. Lynch WJ, Carroll ME. Sex differences in the acquisition of intravenously selfadministered cocaine and heroin in rats. Psychopharmacol (Berl). 1999;144:77-82.

11. Perry AN, Westenbroek C, Becker JB. The development of a preference for cocaine over food identifies individual rats with addiction-like behaviors. PLoS ONE. 2013;8:e79465.

12. Roberts DCS, Bennett SAL, Vickers GJ. The estrous cycle affects cocaine selfadministration on a progressive ratio schedule in rats. Psychopharmacol (Berl). 1989;98:408-11.

13. Roth ME, Carroll ME. Sex differences in the escalation of intravenous cocaine intake following long- or short-access to cocaine self-administration. Pharmacol Biochem Behav. 2004;78:199-207.

14. Anker JJ, Carroll ME. Females are more vulnerable to drug abuse than males: Evidence from preclinical studies and the role of ovarian hormones. Curr Top Behav Neurosci. 2010;8:73-96.

15. Becker JB, Koob GF. Sex Differences in Animal Models: Focus on Addiction. Pharmacol Rev. 2016;68:242-63.

16. Hu M, Crombag HS, Robinson TE, Becker JB. Biological basis of sex differences in the propensity to self-administer cocaine. Neuropsychopharmacology . 2004;29:81-85.

17. Dews P. Modification by Drugs of Performance on Simple Schedules of Positive Reinforcement. 1956. 1956.

18. Miczek KA, Grossman SP. Positive conditioned suppression: Effects of CS duration. J Exp Anal Behav. 1971;15:243-7.

19. Pickens R, Thompson T. Cocaine-reinforced behavior in rats: Effects of reinforcement magnitude and fixed-ratio size. J Pharmacol Exp Ther. 1968;161:122-9.

20. Schuster CR, Thompson T. Self administration of and behavioral dependence on drugs. Annu Rev Pharmacol. 1969;9:483-502.

21. Tornatzky W, Miczek KA. Cocaine self-administration 'binges': Transition from behavioral and autonomic regulation toward homeostatic dysregulation in rats. Psychopharmacol (Berl). 2000;148:289-98.

22. Becker JB. Sex differences in addiction. Dialog- Clin Neurosci. 2016;18:395-402.

23. Evans SM, Haney M, Foltin RW. The effects of smoked cocaine during the follicular and luteal phases of the menstrual cycle in women. Psychopharmacol (Berl). 2002;159:397-406.

24. Evans SM, Foltin RW. Does the response to cocaine differ as a function of sex or hormonal status in human and non-human primates? Horm Behav. 2010;58:13-21.
25. Hecht GS, Spear NE, Spear LP. Changes in Progressive Ratio Responding for Intravenous Cocaine throughout the Reproductive Process in. 1999.

26. Calipari ES, Juarez B, Morel C, Walker DM, Cahill ME, Ribeiro E, et al. Dopaminergic dynamics underlying sex-specific cocaine reward. Nat Commun. 2017; 8:1-15.

27. Cartoni E, Balleine B, Baldassarre G. Appetitive pavlovian-instrumental transfer: a review. Neurosci Biobehav Rev. 2016;71:829-48.

28. Lamb RJ, Schindler CW, Pinkston JW. Conditioned stimuli's role in relapse: preclinical research on Pavlovian-Instrumental-Transfer. Psychopharmacol (Berl). 2016;233:1933-44.

29. Calipari ES, Ferris MJ, Jones SR. Extended access of cocaine self-administration results in tolerance to the dopamine-elevating and locomotor-stimulating effects of cocaine. J Neurochem. 2014;128:224-32.

30. Calipari ES, Godino A, Peck EG, Salery M, Mervosh NL, Landry JA, et al. Granulocyte-colony stimulating factor controls neural and behavioral plasticity in response to cocaine. Nat Commun. 2018;9:9.

31. Caligioni CS. Assessing reproductive status/stages in mice. Curr Protoc Neurosci. 2009;Appendix 4:Appendix4I.

32. Oleson EB, Roberts DCS. Behavioral economic assessment of price and cocaine consumption following self-administration histories that produce escalation of either final ratios or intake. Neuropsychopharmacology. 2009; 34:796-804.

33. Siciliano CA, Jones SR. Cocaine potency at the dopamine transporter tracks discrete motivational states during cocaine self-administration. Neuropsychopharmacology . 2017;42:1893-904.

34. Oleson EB, Richardson JM, Roberts DCS. A novel IV cocaine self-administration procedure in rats: differential effects of dopamine, serotonin, and GABA drug pretreatments on cocaine consumption and maximal price paid. Psychopharmacol (Berl). 2011;214:567-77.

35. Oleson EB, Roberts DCS. Cocaine Self-Administration in Rats: Threshold Procedures. Methods Mol Biol. 2012;829:303-19.

36. Christensen CJ, Silberberg A, Hursh SR, Roma PG, Riley AL. Demand for cocaine and food over time. Pharmacol Biochem Behav. 2008;91:209-16.

37. Hursh SR, Silberberg A. Economic demand and essential value. Psychol Rev. 2008;115:186-98.

38. Hursh SR, Winger G. Normalized demand for drugs and other reinforcers. J Exp Anal Behav. 1995;64:373-84.

39. Grimm JW, Hope BT, Wise RA, Shaham Y. Neuroadaptation. Incubation cocaine craving withdrawal Nat. 2001;412:141-2.

40. LeBlanc KH, Ostlund SB, Maidment NT. Pavlovian-to-instrumental transfer in cocaine seeking rats. Behav Neurosci. 2012;126:681-9.

41. Ciccocioppo R, Sanna PP, Weiss F. Cocaine-predictive stimulus induces drugseeking behavior and neural activation in limbic brain regions after multiple months of abstinence: reversal by $\mathrm{D}(1)$ antagonists. Proc Natl Acad Sci USA. 2001;98:1976-81.

42. Kalivas PW, McFarland K. Brain circuitry and the reinstatement of cocaine-seeking behavior. Psychopharmacol (Berl). 2003;168:44-56.

43. LaLumiere RT, Smith KC, Kalivas PW. Neural circuit competition in cocaineseeking: roles of the infralimbic cortex and nucleus accumbens shell. Eur J Neurosci. 2012;35:614-22.

44. Lacy RT, Strickland JC, Feinstein MA, Robinson AM, Smith MA. The effects of sex, estrous cycle, and social contact on cocaine and heroin self-administration in rats. Psychopharmacol (Berl). 2016;233:3201-10.

45. Richardson NR, Roberts DCS. Progressive ratio schedules in drug selfadministration studies in rats: a method to evaluate reinforcing efficacy. J Neurosci Methods. 1996;66:1-11.

46. Bickel WK, Green L, Vuchinich RE. Behavioral economics. J Exp Anal Behav. 1995;64:257-62.

47. Robinson AM, Fronk GE, Zhang $H$, Tonidandel S, Smith MA. The effects of social contact on cocaine intake in female rats. Drug Alcohol Depend. 2017;177:48-53.

48. Saunders BT, Richard JM, Margolis EB, Janak PH. Dopamine neurons create Pavlovian conditioned stimuli with circuit-defined motivational properties. Nat Neurosci. 2018;21:1072-83.

49. Anderson SM, Bari AA, Pierce RC. Administration of the D1-like dopamine receptor antagonist $\mathrm{SCH}-23390$ into the medial nucleus accumbens shell attenuates cocaine priming-induced reinstatement of drug-seeking behavior in rats. Psychopharmacol (Berl). 2003;168:132-8.

50. Calipari ES, Bagot RC, Purushothaman I, Davidson TJ, Yorgason JT, Peña CJ, et al. In vivo imaging identifies temporal signature of D1 and D2 medium spiny neurons in cocaine reward. Proc Natl Acad Sci USA. 2016;113:2726-31.

51. Dackis CA, Gold MS. New concepts in cocaine addiction: The dopamine depletion hypothesis. Neurosci Biobehav Rev. 1985;9:469-77.

52. Hummel M, Unterwald EM. D1 dopamine receptor: A putative neurochemical and behavioral link to cocaine action. J Cell Physiol. 2002;191:17-27. 